\title{
PROCESO DE INVESTIGACIÓN DE MERCADOS
}

Responsable: Lic. Adm. Ruperto Layme Uchochoque

\section{RESUMEN}

El presente artículo es un documento que nos permite conocer qué es la investigación de mercados y los conceptos más modernos al respecto en este momento. Ponemos al alcance de lectores deseosos en querer aprender a realizar una investigación de mercados para cualquier giro o tipo de negocio, y así evitar trabajar informalmente, ya que el mercado en su conjunto ha evolucionado raudamente y es necesario mantenerlos en permanente dinámica y progreso de la empresa de su interés.

\section{ABSTRACT}

Text the present denominated "Process of Investigation of markets" is a document that allows us to know that it is the most modern investigation of markets and concepts at this moment. We put within reach of generous readers in wanting to learn to make an investigation of markets for any turn or type of business and thus of avoiding to work informally since the evolved market as a whole to raudamente, and is necessary to maintain in permanent dynamics and progress of the company of its interest.

\section{LA INVESTIGACIÓN DE MERCADO}

La investigación de mercados es la recopilación, registro y análisis sistemático de datos relacionados con problemas de mercado de bienes y servicios: Para nuestros fines, hay cuatro términos que tenemos que considerar: sistemático, objetivo, informativo y toma decisiones. Por consiguiente, nosotros definimos investigación de mercados como un enfoque sistemático y objetivo hacia el desarrollo y provisión de información aplicable al proceso de toma de decisiones en la gerencia de mercadeo.

Lo de sistemático se refiere a la necesidad de que el proyecto de investigación esté bien organizado y planeado. La objetividad implica que la investigación de mercados se esfuerza por ser imparcial e insensible en la realización de sus responsabilidades.

\section{PROCESO DE INVESTIGACIÓN DE MERCADOTECNIA}

El proceso de investigación de mercado contiene los siguientes pasos:

\section{Establecer la Necesidad de Información}

Para elaborar la investigación de mercados, puede un gerente establecer la necesidad de investigación. El investigador debe entender, claramente, la razón por la cual se necesita la información. Se debe establecer la necesidad de determinada información sobre la investigación, es una de las fases críticas y difíciles del proceso de investigación.

La definición del problema de investigación de mercados está determinada por las correcciones que se desean realizar en la empresa de bienes 0 servicios, es decir, se deben solucionar los problemas de la empresa hacia fuera. ¿Cómo determinar el problema? cuál es la información necesaria y cómo puede obtenerla de manera eficiente y eficaz.

\section{Especificar los Objetivos de la Investigación}

El objetivo de investigación de mercados consiste en proporcionar información obtenida de investigaciones profundas para la toma de decisiones. Esta información específica que se requiere es para resolver los problemas de investigación de mercados. Los objetivos responden a la pregunta "¿Por qué se está llevando a cabo este proyecto?" Comúnmente se establecen por escrito. Las necesidades de información deben responder a la pregunta "¿Por qué se necesita una información específica para poder lograr los objetivos?" Puede considerarse como una enumeración detallada de los objetivos de la investigación.

Decidir respecto a los objetivos de investigación requiere comprender el tipo de investigación que se está realizando. Comprende desde lo explorativo (que asume que no se tiene ningún concepto preconcebido) hasta lo causal (que asume formas específicas de cómo una o más variables influyen en una o más de las otras variables, y lo descriptivo (que asume describir las características de la población o fenómeno que se estudia. Que responda a preguntas como ¿Qué?, ¿cómo?, ¿dónde?, ¿por qué? y ¿cuando?).

Si una empresa quiere saber acerca del porqué unos de sus productos ha aumentado o disminuido sus ventas, lanzar un nuevo producto 
al mercado, reposicionar una marca, modificar el envase o diseñar la campaña de comunicación todo ello, debe de responder a una estrategia de mercadotecnia. Lo único que se va a obtener de la investigación es información que será otorgada al gerente de mercadotecnia para que tome las decisiones que le permitan cumplir con los objetivos.

La investigación preliminar es la recopilación de información de antecedentes del negocio y de su medio ambiente, a través de entrevistas con personas ajenas a la compañia como puede ser un consumidor. La investigación preliminar consiste en obtener las opiniones acerca de los productos o servicios en el mercado y las condiciones en que se encuentran en pequeña escala preferentemente.

La hipótesis es una suposición o teoria que un investigador o gerente formula acerca de una característica de la población en estudio susceptible de ser comprobada. Estas hipótesis deben de ser aceptadas o rechazadas cuando se concluya la investigación.

El diseño de la Investigación es el plan que se va a seguir para responder o cumplir los objetivos o hipótesis de de investigación de mercado, estructura o marco para resolver un problema especifico. Es decir, guía la recolección de datos y analiza las etapas del proyecto de investigación. Especifica el tipo de información a ser recolectada, las fuentes de datos, los procedimientos y análisis de recolección de datos. Una vez planteado, se someterá a la aprobación de la empresa.

\section{Determinar las Fuentes de Datos}

El siguiente paso es determinar si los datos pueden conseguirse actualizados de las fuentes internas o externas de la organización. Las fuentes internas contienen los estudios previos de investigación y los antecedentes de la empresa. Las fuentes externas incluyen informes comerciales de investigación, revistas de negocios, informes gubernamentales, etc. Si los datos no están disponibles, el siguiente paso será recopilar nuevos datos por medio de entrevistas por correo, telefónicas y personales, observación, experimentación o simulación.

El gerente puede usar diversas fuentes para obtener la información necesaria, por ejemplo cuestionarios, cintas grabadas, entre otros.

\section{Desarrollar las Formas para Recopilar los Datos}

El contenido dependerá de que los datos se recopilen por medio de entrevistas o de la observación. El proceso mediante el cual se desarrollan los formularios para recopilación de datos por medio de entrevistas es más complejo; la redacción de las preguntas, la secuencia de las preguntas, la utilización de preguntas directas en contraposición a las indirectas y el formato en general del cuestionario son muy importantes.

La fase de recopilación es una oportunidad de intentar o probar y seleccionar los procedimientos necesarios cuando los datos se tengan disponibles.

\section{Diseñar la Muestra}

Se tiene que definir la población o universo de interés, pues de este grupo se tomará la muestra. Después de definir la muestra, saber si se va a emplear: la muestra probabilística, cada elemento de la población tiene una oportunidad conocida y diferente de cero de ser seleccionado. Las muestras no probabilísticas incluyen un muestreo aleatorio simple, un muestreo en grupo y un muestreo estratificado. El tercer asunto se refiere al tamaño de la muestra. El tamaño apropiado de la muestra depende de muchas consideraciones. Estas oscilan desde fómulas estadísticas precisas para determinar el tamaño de la muestra hasta consideraciones generales relacionadas con el costo, el valor y la exactitud de la información necesaria, para la toma de decisiones.

Estadísticamente, la representatividad de la muestra exige que todos los componentes del universo tengan la misma probabilidad de figurar en la muestra. Respetando este principio, se citan algunos criterios de elección de muestras:

1) Método de las muestras por azar:

Se trata de sortear entre todos los componentes del universo aquellas personas que van a formar parte de la muestra calculada.

2) Método de las cuotas estratificadas:

Consiste en asignar unas determinadas cuotas a los entrevistadores en las que se concretan las condiciones de las personas a entrevistar.

3) Método de las muestras mixtas:

Se trata de aplicar diversos criterios al mismo tiempo en un universo, por ejemplo mezclando los criterios geográficos de ámbito nacional y las zonas rurales $y$ urbanas.

\section{Recopilar los Datos}

Es crítico ya que, generalmente, comprende una proporción grande del presupuesto destinado a la investigación y una gran porción del error total en los resultados de la investigación. La selección, el entrenamiento y el control de los entrevistadores son esenciales en los estudios efectivos de investigación de mercados.

\section{Procesar los Datos}

Una vez que se han registrado los datos, comienza su procesamiento manual y computacional. Esto incluye: La edición comprende el repaso de los formularios en los cuales se han recopilado los datos en relación con 
la legibilidad, consistencia y su totalidad. La codificación abarca desde categorias por respuestas o grupos de respuestas, con el fin de que puedan utilizarse numerales para representar las categorias.

\section{Analizar los Datos.}

Hay tres formas de análisis: el univariado se refiere al examen de cada una de las variables, por separado. El objetivo es lograr una descripción más concienzuda del grupo de datos; el bivariado se refiere a la relación que existe entre dos variables, mientras que el análisis multivariado comprende el análisis simultáneo de tres o más variables. El objetivo del análisis bivariado y el multivariado es primordialmente explicativo.

\section{Presentar los Resultados de la Investigación.}

Una vez terminado el análisis de datos, el investigador prepara el informe y comunica las conclusiones y recomendaciones a la gerencia. Es un informe claro, preciso y conciso por escrito y una presentación oral simple y completa. EI resultado debe presentarse en un formato sencillo y enfocado hacia las necesidades de información de la situación de decisión.

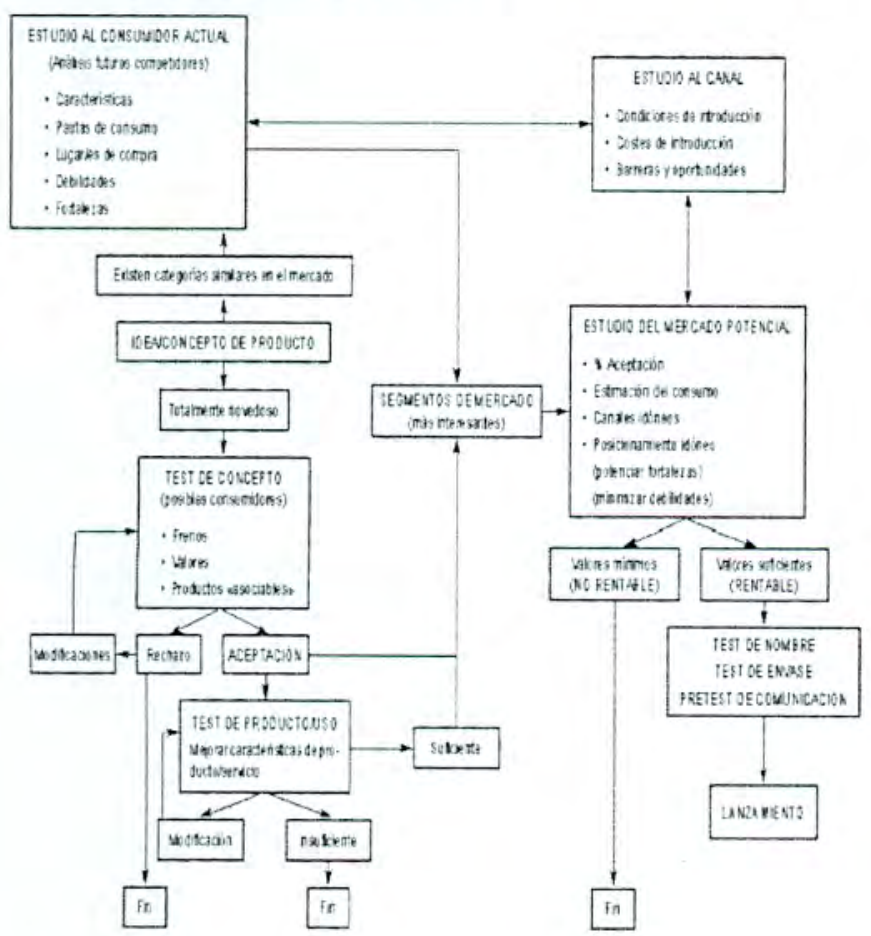

GRÁFICO: Esquema de viabilidad y desarrollo de un producto

III. ANEXOS: (Ver esquemay otros)

\section{Muestreo Aleatorio}

Explicación gráfica:

Coeficiente de elevación $\mathrm{Ce}=\frac{N}{n}$
A continuación se elige al azar un número entre la unidad y el $\mathrm{Ce}$.

1 ,

$1^{\circ}$ seleccionado $=x$

$2^{\circ}$ seleccionado $=\mathrm{x}+\mathrm{Ce}$

$3^{\circ}$ seleccionado $=2 .^{\circ}+\mathrm{Ce}$

$4^{\circ}$ seleccionado $=3 .^{\circ}+\mathrm{Ce}$

$5^{\circ}$ seleccionado $=4 .^{\circ}+\mathrm{Ce}$

\section{Tamaño de la Muestra}

\section{Fórmulas Generales:}

- Para poblaciones finitas (menos de 100000 habitantes):

$$
n=\frac{Z^{2} x P x Q}{E^{2}}
$$

- Para poblaciones finitas (menos de 100000 habitantes):

$$
n=\frac{Z^{2} x P x Q x N}{E^{2}(N-1)+Z^{2} x P / Q}
$$

\section{Leyenda:}

$\mathbf{n}$ = Número de elementos de la muestra.

$\mathbf{N}=$ Número de elementos del universo.

$P / Q=$ Probabilidades con las que se presenta el fenómeno.

$\mathbf{Z}^{2}=$ Valor crítico correspondiente al nivel de confianza elegido; siempre se opera con valor sigma 2 , luego $Z=2$.

$\mathbf{E}=$ Margen de error permitido (a determinar por el investigador).

\section{BIBLIOGRAFÍA:}

Etzel/Stanton/Warkel. Fundamentos de marketing. Editorial Mc Graw Hill, 1999,Mexico.

Jany C. José MN. Investigación integral de mercados. Editorial Mc. Graw Hill,1994,Colombia Jeffrey Pope. Investigación de mercados Norma,1984, Colombia.

Kinnear T.,TaylorJ. Investigación de mercados un enfoque aplicativo. Editorial Mc Graw Hill. tercera edición, 1991,Mexico.

Malhotra Naresh. Investigación de mercados un enfoque práctico. Editorial Prentice Hall Hispanoamericana, segunda edición,1997, Mexico.

Weiers Ronald M. Investigación de mercados. Editorial Prentice Hall Hispanoamericana, 1086, Mexico. 
V. CASO PRÁCTICO: EMPRESA DE TRANSPORTES "FLORES Hnos." (Sólo resumen)
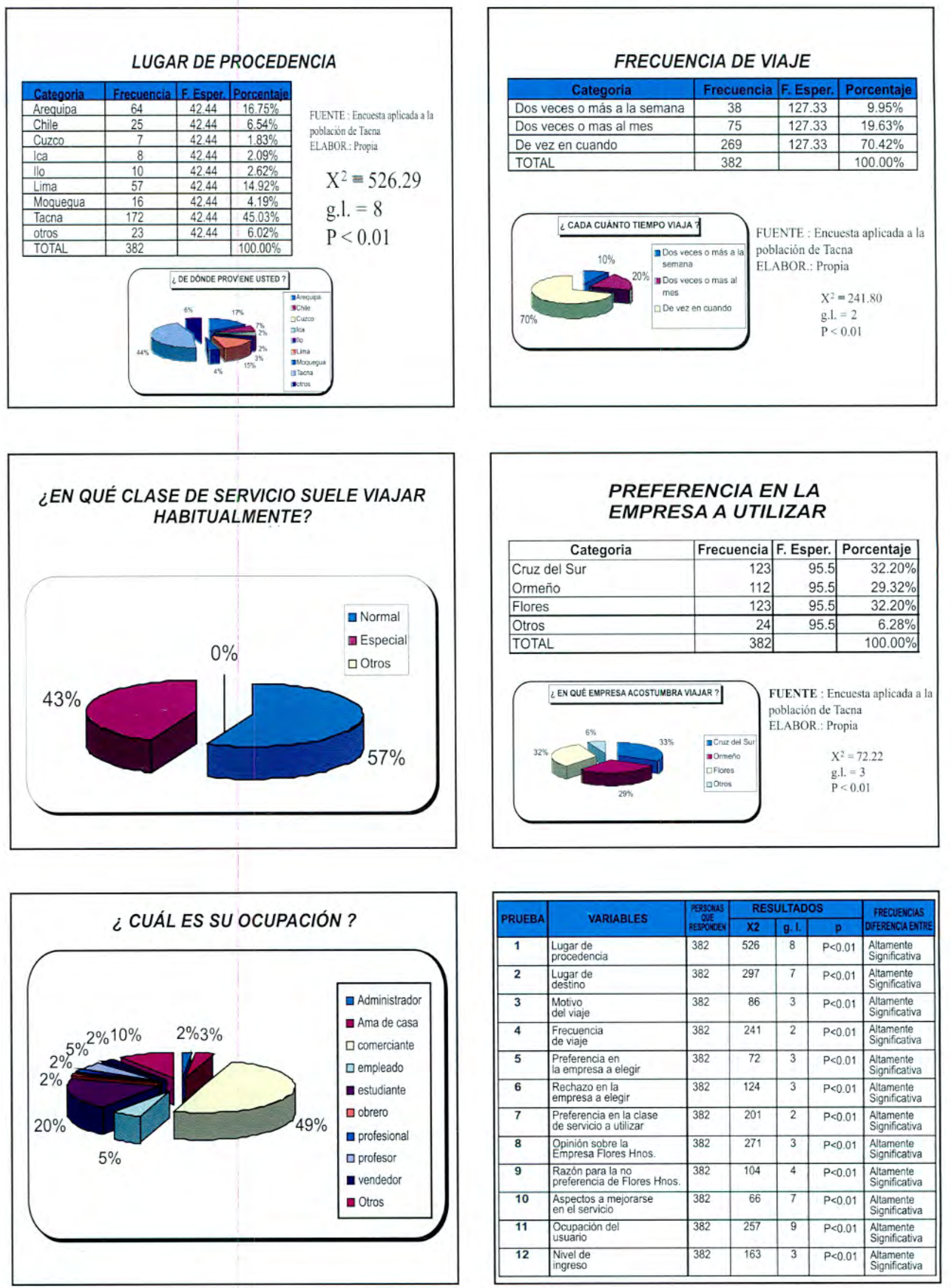

\begin{tabular}{|c|c|c|c|c|c|c|}
\hline \multirow{2}{*}{ PRUEBA } & \multirow{2}{*}{ VARIABLES } & \multirow{2}{*}{ 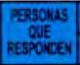 } & \multicolumn{3}{|c|}{ RESULTADOS } & \multirow{2}{*}{\begin{tabular}{|c|} 
FRECUENCIAS \\
DFERENCAEMTRE
\end{tabular}} \\
\hline & & & $x_{2}$ & g. 1. & p & \\
\hline 1 & $\begin{array}{l}\text { Lugar de } \\
\text { procedencia }\end{array}$ & 382 & 526 & 8 & $P<0.01$ & \begin{tabular}{|l|} 
Altamente \\
Significativa \\
\end{tabular} \\
\hline 2 & $\begin{array}{l}\text { Lugar de } \\
\text { destino }\end{array}$ & 382 & 297 & 7 & $P<0.01$ & \begin{tabular}{|l} 
Altamente \\
Significativa
\end{tabular} \\
\hline 3 & \begin{tabular}{|l|} 
Motivo \\
del viaje
\end{tabular} & 382 & 86 & 3 & $P<0.01$ & \begin{tabular}{|l|} 
Altamente \\
Significativa \\
\end{tabular} \\
\hline 4 & \begin{tabular}{|l}
$\begin{array}{l}\text { Frecuencia } \\
\text { de viaje }\end{array}$ \\
\end{tabular} & 382 & 241 & 2 & $P<0.01$ & \begin{tabular}{|l} 
Altamente \\
Significativa \\
\end{tabular} \\
\hline 5 & \begin{tabular}{|l|} 
Preferencia en \\
la empresa a elegir \\
\end{tabular} & 382 & 72 & 3 & $P<0.01$ & \begin{tabular}{|l|} 
Altamente \\
Significativa \\
\end{tabular} \\
\hline 6 & $\begin{array}{l}\text { Rechazo en la } \\
\text { empresa a elegir }\end{array}$ & 382 & 124 & 3 & $P<0.01$ & \begin{tabular}{|l} 
Altamente \\
Significativa \\
\end{tabular} \\
\hline 7 & $\begin{array}{l}\text { Preferencia en la clase } \\
\text { de servicio a utilizar }\end{array}$ & 382 & 201 & 2 & $P<0.01$ & \begin{tabular}{|l|} 
Altamente \\
Significativa \\
\end{tabular} \\
\hline 8 & $\begin{array}{l}\text { Opinión sobre la } \\
\text { Empresa Flores Hnos. }\end{array}$ & 382 & 271 & 3 & $P<0.01$ & \begin{tabular}{|l|} 
Allamente \\
Significativa \\
\end{tabular} \\
\hline 9 & $\begin{array}{l}\text { Razón para la no } \\
\text { preferencia de Flores Hnos. }\end{array}$ & 382 & 104 & 4 & $P<0.01$ & \begin{tabular}{|l|} 
Altamente \\
Significativa \\
\end{tabular} \\
\hline 10 & $\begin{array}{l}\text { Aspectos a mejorarse } \\
\text { en el servicio }\end{array}$ & 382 & 66 & 7 & $P<0.01$ & \begin{tabular}{|l|} 
Altamente \\
Significativa \\
\end{tabular} \\
\hline 11 & $\begin{array}{l}\text { Ocupación del } \\
\text { usuario }\end{array}$ & 382 & 257 & 9 & $P<0.01$ & \begin{tabular}{|l|} 
Altamente \\
Significativa \\
\end{tabular} \\
\hline 12 & \begin{tabular}{|l} 
Nivel de \\
ingreso
\end{tabular} & 382 & 163 & 3 & $P<0.01$ & \begin{tabular}{|l} 
Altamente \\
Significativa
\end{tabular} \\
\hline
\end{tabular}

